\title{
Anaphore et conjugaison objective en mordve
}

Conclusion et perspectives

\section{Arnaud Fournet}

\section{(2) OpenEdition}

\section{Journals}

Édition électronique

URL : https://journals.openedition.org/efo/4175

DOI : $10.4000 /$ efo. 4175

ISSN : 2275-1947

Éditeur

INALCO

Édition imprimée

Date de publication : 1 janvier 2014

ISBN : 978-2-343-05394-3

ISSN : 0071-2051

Référence électronique

Arnaud Fournet, «Anaphore et conjugaison objective en mordve », Études finno-ougriennes [En ligne], 46 | 2014, mis en ligne le 14 octobre 2015, consulté le 08 juillet 2021. URL : http:// journals.openedition.org/efo/4175; DOI : https://doi.org/10.4000/efo.4175

Ce document a été généré automatiquement le 8 juillet 2021

\section{(c) (i) (3)}

Études finno-ougriennes est mis à disposition selon les termes de la Licence Creative Commons Attribution - Pas d'Utilisation Commerciale 4.0 International. 


\title{
Anaphore et conjugaison objective en mordve
}

\author{
Conclusion et perspectives
}

Arnaud Fournet

1 L'article s'intéresse à la double série de désinences verbales qui existe en mordve suivant que le complément d'objet est défini ou indéfini, ce que la tradition ouralistique appelle conjugaison objective définie, ou peut-être faudrait-il mieux dire à objet défini (CàOD), et d'autre part conjugaison objective indéfinie, à savoir à objet indéfini (CàOI). Toutes les langues ouraliennes ne présentent pas cette distinction et se pose donc la question de l'origine de ces formes. Ce phénomène morphologique, qui implique en même temps une composante sémantico-syntaxique, existe également en hongrois où il résulte d'une innovation, à notre avis tout à fait indépendante de ce qui peut s'observer en mordve. Le présent article proposé à la sagacité érudite des ouralicistes décrit en premier lieu le corpus et le jeu des suffixes pronominaux en mokcha, en comparaison avec ceux de l'erza, sa langue sœur la plus proche, et s'efforce de démonter les suffixes des CàOD et CàOI afin d'en déterminer les composants et morphèmes élémentaires. Dans un deuxième temps, une hypothèse inédite, ou en tout cas dont nous n'avons pas trouvé de précurseur dans la littérature, est développée pour expliquer l'émergence de la dualité CàOD et CàOI en mordve. Par ailleurs, il convient de souligner que nous ne prétendons pas dans cet article établir un état des lieux critique, et encore moins exhaustif, de la bibliographie relative à la problématique des CàOD et CàOI en mordve, bien qu'il soit fait au passage quelques observations, et à ce sujet nous pensons en particulier à l'approche naguère défendue par J.Perrot sur cette question de l'émergence la dualité CàOD et CàOI. Comme il apparaîtra dans la suite, nous ne croyons pas que l'origine des CàOD et CàOI en mordve ait un lien avec les pronoms possessifs, comme J. Perrot l'avait proposé. 


\section{Introduction}

Plusieurs langues ouraliennes présentent ce que la tradition appelle des conjugaisons objectives définie et indéfinie. Nous commençons l'article par la description de ce phénomène en prenant l'exemple du mokcha, une langue ouralienne parlée en Russie à quelque cinq cents kilomètres au sud-est de Moscou et formant avec son proche parent, l'erza, l'ensemble mordve.

3 Le plus simple, sans doute, pour introduire les notions de CàOD ou CàOI, est de recourir à une langue comme le français. Ainsi on peut comparer : il mange de la viande il mange la viande. Le premier énoncé est un exemple de CàOI et le suivant de CàOD: le complément d'objet y est déterminé par l'article défini. En français, la présence de l'article défini n'a toutefois aucune incidence sur la forme du verbe, qui est la même dans les deux cas. Cet exemple se traduit en mokcha de la façon suivante : сон ярхцай сиволь сон ярхцасы сивольть. Ces énoncés se laissent analyser comme suit :

- /son/ < $\mathrm{COH}>$ 'P3 au cas nominatif' est le sujet grammatical, correspondant à 'il, elle',

- /jařtsa/ <ярхца> 'manger', un verbe, est le centre de l'énoncé,

- /sivəl/ <сиволь> 'viande', un nom, est le complément d'objet,

- /t/ <ть> 'article défini au cas accusatif-génitif', détermine /sivəl/ <сиволь> 'viande'.

4 Le point remarquable est que le verbe /jařtsa/ <ярхца> est tantôt suffixé par /sì / <сы> (CàOD) tantôt par $/ \mathrm{j} /<\breve{\mathrm{h}>}($ CàOI), suivant que l'objet soit défini ou non. C'est le principe de fonctionnement suffixal des CàOD et CàOI en ouralien: il existe deux séries de suffixes pour les verbes suivant que l'objet est défini ou non. Signalons au passage que / jařtsaj/ <ярхцай> 'il mange' et /jařtsasi/ <ярхцасы> 'il le/la mange' sont deux phrases acceptables. Le pronom /son/ $<\mathrm{COH}>$, sujet du verbe, n'est pas obligatoire pour former une phrase complète.

5 Avant de formuler une hypothèse sur la genèse des CàOD et CàOI en mordve, il convient d'abord d'en dresser le tableau des formes tant littéraires que dialectales.

\section{La prédication nominale}

Les signifiants des pronoms personnels au nominatif sont les suivants en mokcha : P1 /

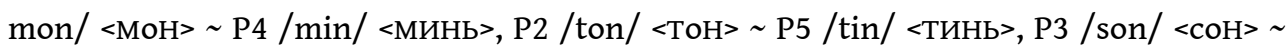
$\mathrm{P} 6 / \sin /<$ синь>. On peut noter le parallélisme des formes entre le singulier et le pluriel. Le pluriel des noms au nominatif est obtenu en suffixant $/ \mathrm{t} /<\mathrm{T}(\mathrm{b})>$ : /jalga/ <ялга> 'ami(e)' /jalgat/ <ялгат> 'ami(e)s'. Ce nom commun est épicène, car il n'y a pas de genre grammatical en mokcha. Par ailleurs nous laissons de côté les questions graphiques relatives à la distribution de $<\mathrm{T}>\mathrm{et}\langle\mathrm{Tb}\rangle$.

7 Il n'y a pas de copule au présent pour la prédication nominale : /son jalga/ <coн ялга> 'il, elle est un(e) ami(e)', /sin jalgat/ <синь ялгат> 'ils, elles sont des ami(e)s'. De ce fait, le suffixe /-t/ est à la fois : (1) le signifiant du pluriel des noms et (2) le signifiant de 'P6 au présent'. On peut observer qu'un certain nombre de suffixes présentent une perméabilité entre noms et verbes. C'est une tendance générale en mokcha, voire en mordve, que la forme relative à $\mathrm{P} 6$ ou P3 sert de plaque tournante morphologique par laquelle des suffixes (pro)nominaux à l'origine se trouvent être utilisés pour les verbes avec diverses réinterprétations fonctionnelles. Ce point, qui sera développé dans la 
suite chaque fois que nécessaire, éclaire en filigrane l'hypothèse proposée à la fin de la description.

Le paradigme complet du présent est le suivant : /jalgan/ <ялган> 'je suis un(e) ami(e)', /jalgat/ <ялгат> 'tu es un(e) ami(e)', /jalga/ <ялга> 'il, elle est un(e) ami(e)', / jalgatama/ <ялгатама> 'nous sommes des ami(e)s', /jalgatada/ <ялгатада> 'vous êtes des ami(e)s', /jalgat/ <ялгат> 'ils, elles sont des ami(e)s'.

Il existe également un imparfait, appelé омьоце ётай пингсь ou deuxième passé par Aliamkin (2000, p.127): /jalgalen/ <ялгалень> 'j'étais un(e) ami(e)', /jalgalet/ <ялгалет> 'tu étais un(e) ami(e)', /jalgal/ <ялгаль> 'il, elle était un(e) ami(e)', / jalgaleme/ <ялгалеме> 'nous étions des ami(e)s', /jalgalede/ <ялгаледе> 'vous étiez des ami(e)s', /jalgadt/ <ялгальхть> 'ils, elles étaient des ami(e)s'. Le suffixe du pluriel provoque certaines alternances phonémiques, ce qui explique qu'on ait $/ \mathrm{l} /<\pi \mathrm{b}>$ dans / jalgal/ mais / $\mathrm{A} /<\mathrm{Jxb}>$ dans /jalgadt/. Ce suffixe était autrefois $* / \mathrm{ht} /$, le /t/ était précédé d'une fricative vélaire sourde, encore prononcée comme telle dans certains dialectes mokchas. La plus ancienne description du mokcha, Ahlquist (1861), n'a d'ailleurs que la forme $/ \mathrm{ht} /$.

10 Le signifiant de l'imparfait est /-1-/ <л(ь)> et il possède la propriété de pouvoir se suffixer à un nom, un verbe, un adjectif et à presque toutes les classes syntaxiques, hormis les adverbes et les conjonctions.

11 Le premier jeu de suffixes, que nous venons d'identifier, peut être résumé dans le tableau suivant :

Tableau 1. Suffixes de la prédication nominale

\begin{tabular}{|l|l|l|l|l|l|l|}
\hline & P1 & P2 & P3 & P4 & P5 & P6 \\
\hline Pluriel & & & & & & \multirow{2}{*}{ - $<*$ ht } \\
\hline Présent & $-\mathrm{n}$ & $-\mathrm{t}$ & $-\varnothing$ & -t-ama & -t-ada & \\
\hline Imparfait & -le-n & - le-t & $-1-\varnothing$ & - l-eme & -l-ede & -4-t $<*$ lht \\
\hline
\end{tabular}

\section{Les suffixes de la CàOI}

Les verbes employés de façon intransitive (donc sans aucun objet) ou bien transitive à la CàOI (avec un objet indéterminé) reçoivent pour l'essentiel les suffixes précédemment décrits. Nous illustrons les paradigmes avec le verbe /jaka/ <яка> 'marcher' au présent et à l'imparfait: /jakan/ <якан> 'je marche' /jakalen/ <якалень> 'je marchais', /jakat/ <якат> 'tu marches' /jakalet/ <якалеть> 'tu marchais', /jakaj/ <якай> 'il, elle marche' /jakas/ <якась> 'il, elle marchait', / jakatama/ <якатама> 'nous marchons' /jakaleme/ <якалеме> 'nous marchions', / jakatada/ <якатада> 'vous marchez' /jakalede/ <якаледе> 'vous marchiez', /jakaçt/ <якайхть> 'ils, elles marchent' /jaka(j)st/ <якасть> 'ils, elles marchaient'.

Les suffixes sont en partie communs avec les paradigmes que nous avons décrits au paragraphe précédent. C'est le cas pour P1, P2, P4 et P5. Par ailleurs, la forme de présent /jakaj/ <якай> 'il, elle marche' est homophone de celle du participe présent / jakaj/ <якай> 'marchant, marcheur'. /son jakaj/ <сон якай> signifie à la fois 'il, elle 
marche' et 'il, elle est marcheur, marchant, en train de marcher'. /jakaçt/ <якайхть> est de fait un participe pluralisé par le suffixe de 'Pluriel nominal' /-(h)t/. Notons que certains dialectes ont conservé l'ancienne forme /jakajht/, où l'alternance $/ \mathrm{j} / \sim / c ̧ /$ est parallèle à celle de $/ 1 /$ / $\$$ / examinée plus haut.

Poursuivons la description avec le paradigme du prétérit: /jakajn/ <якань> 'j'ai marché,, /jakat/ <якать> 'tu as marché', /jakas/ <якась> 'il, elle a marché' (syncrétique de l'imparfait), /jakame/ <якаме> 'nous avons marché', /jakade/ <якаде> 'vous avez marché', /jaka(j)st/ <якасть> 'ils, elles ont marché' (syncrétique de l'imparfait). Ce temps est appelé premier passé (васенце ётай пингсь) par Aljamkin (2000, p. 127).

Cette série de suffixes du prétérit ne peut pas être affixée à un signifiant nominal, ce qui atteste l'existence d'une opposition verbo-nominale en mokcha, même s'il est facile d'observer qu'un certain nombre d'autres suffixes sont en quelque sorte mutualisés entre noms et verbes, comme nous l'avons souligné précédemment.

Finissons ce chapitre descriptif en soulignant que la base verbale /jaka/ <яка> ne peut jamais être employé nue sans suffixe. Même à l'impératif, les verbes sont suffixés, ainsi : /jakak/ <якак> 'marche!' /jakada/ <якада> 'marchez!'. Concernant la forme $\mathrm{du}$ singulier, on peut noter que le suffixe /-k/ n'a pas de rapport avec /ton/ $<\mathrm{TOH}>\mathrm{P} 2$, en outre ce suffixe présente une allomorphie :/-k/ ou /-t/ suivant que la base verbale finit par une voyelle ou une consonne respectivement. Quant à P5 le suffixe est /-da/ ou /-ada/ dans les mêmes conditions.

17 L'indicatif ne comprend pas d'autres temps simples que le présent, l'imparfait et le prétérit. Il existe un futur périphrastique construit avec le verbe /karma/ <карма> 'commencer' au présent : /karma-n jakama/ <карман якама> 'je marcherai', /karma-t jakama/ <кармат якама> 'tu marcheras', etc. Ce temps n'a pas de singularité particulière en ce qui concerne les suffixes qui sont entièrement prévisibles.

18 Les différents suffixes déjà rencontrés peuvent être regroupés dans le tableau suivant où sont visibles les syncrétismes et les passerelles entre formes nominales et verbales :

Tableau 2. Suffixes de la prédication nominale et de la CàOI

\begin{tabular}{|c|c|c|c|c|c|c|}
\hline & P1 & P2 & P3 & P4 & P5 & P6 \\
\hline Impératif & & $-\mathrm{k} /-\mathrm{t}$ & & & $-(a) d a$ & \\
\hline \multicolumn{6}{|l|}{ Pluriel (nom.) } & \multirow{2}{*}{$-\mathrm{t}<*$ ht } \\
\hline Présent (nom.) & \multirow{2}{*}{$-n$} & \multirow{2}{*}{$-\mathrm{t}$} & $-\varnothing$ & \multirow{2}{*}{$-t$-ama } & \multirow{2}{*}{-t-ada } & \\
\hline Présent COI & & & $-j$ & & & $-c ̧ t<*$ jht \\
\hline Imparfait (nom.) & \multirow{2}{*}{-le-n } & \multirow{2}{*}{$-1 e-t$} & $-1-\varnothing$ & \multirow{2}{*}{-l-eme } & \multirow{2}{*}{-l-ede } & $-\mathrm{t}-\mathrm{t}<* \mathrm{lht}$ \\
\hline Imparfait COI & & & \multirow{2}{*}{$-s$} & & & \multirow{2}{*}{$-(\mathrm{j}) \mathrm{st}$} \\
\hline Prétérit COI & $-(j) n$ & $-\mathrm{t}$ & & -(e)me & -(e)de & \\
\hline
\end{tabular}

19 Par ailleurs, on peut noter que les suffixes -da, -tada, -lede et -de se laissent aisément comparer avec /tin/ <тинь> P5. La dentale est voisée dans les suffixes, ce qui est un changement phonétique régulier pour les consonnes simples en position intervocalique en mokcha et aussi en erza. A contrario, l'occlusive sourde des suffixes -tama, -tada 
repose non pas sur *-t- mais sur le groupe *-ht-. Nous verrons dans la suite que cette loi permet d'éclairer d'autres suffixes de la CàOD.

\section{La notion de forme pivot}

Dans un système de formes un tant soit peu complexe, il existe toujours des formespivot dont découlent les autres formes de façon prévisible. Cette idée a été développée par un indo-européaniste, Jerzy Kuryłowicz. Elle permet de démonter la genèse progressive d'un système d'après deux principes complémentaires :

- toutes les formes synchroniques contrevenant à la logique interne du système sont susceptibles d'être reformatées en conformité avec les formes attendues,

- une forme qui contrevient à la logique interne est héritée, car elle ne peut pas avoir été créée par le système synchronique, ou bien partiellement héritée car des réaménagements et réalignements partiels sont également possibles.

Dans cette perspective, le jeu de suffixes examiné précédemment peut être analysé de la façon suivante :

Tableau 3. Formes-pivot

\begin{tabular}{|l|l|l|}
\hline & Formes-pivot & Formes dérivées \\
\hline /jalga/ 'ami(e)' & $\Rightarrow>$ /jalga/ 'il, elle est ami(e)' & $\Rightarrow>$ /jalga -n,-t/ \\
\hline /jalgat/ 'ami(e)s' & $\Rightarrow>$ /jalga-t/ 'ils, elles sont ami(e)s' & $\Rightarrow>$ /jalga -t-ama, -t-ada/ \\
\hline /jalga/ 'ami(e)' & $\Rightarrow$ /jalga-l/ 'il, elle était ami(e)' & $\Rightarrow$ /jalga -l-en, -l-et, -l-eme, -l-ede/ \\
\hline /-ht/ Pluriel nom. & $\Rightarrow>$ /-ht/ 'P6 Présent' & $\Rightarrow>$ /-ht-/ 'Présent' (pour P4, P5) \\
\hline
\end{tabular}

On observera la réinterprétation fonctionnelle du suffixe du pluriel nominal /-ht/ qui s'est infiltré dans les suffixes de la conjugaison et qui est devenu synchroniquement le signifiant du présent pour P4 et P5 en opposition avec /-1/, signifiant de l'imparfait.

\section{La problématique de la CàOD}

La référence obligée sur la CàOD en mordve est due à László Keresztes (1999), qui a compilé toutes les formes connues dans l'espace dialectal mokcha et erza. Ce livre va d'ailleurs bien au-delà et compile tous les suffixes verbaux existant dans les dialectes mordves.

Keresztes (1999) discute également les théories proposées pour expliquer l'émergence de toutes ces formes. Le premier chercheur à étudier la CàOD en mordve, Hunfalvy, avait conclu que les suffixes de l'object sont toujours suivis par ceux du sujet. Wiedemann avait atteint la même conclusion. Pour l'essentiel, les approches de la CàOD tendent à proposer des sortes de « gabarits à trous (slots) » avec la structure minimale : Verbe + Objet + Sujet. Elles diffèrent par le nombre de "trous" et l'origine des morphèmes qui viennent les remplir. Les "trous" supplémentaires concernent la notion de temps et de pluriel. Certains chercheurs comme Klemm ou Bubrih avaient pensé que les morphèmes de CàOD étaient de nature nominale et emprunté aux 
pronoms possessifs. Cette idée, que J. Perrot avait reprise ou développée indépendamment, n'explique pas l'essentiel des formes, mais ce phénomène peut avoir joué un rôle dans certains cas comme le montre le tableau 12 et les commentaires qui le suivent.

Une étude détaillée de la CàOD est écrite par Serebrennikov (1967). Il propose d'analyser tous les suffixes de la CàOD d'après un patron général : (Verbe) + Objet + Temps + Sujet. D'après lui les suffixes contemporains remontent initialement à la concaténation en proto-mordve de trois éléments, mais au fil du temps le système serait devenu moins transparent et moins analytique, ce qui expliquerait que les formes actuelles soient souvent assez difficiles à analyser. Un point qui fait difficulté dans l'hypothèse de Serebrennikov est l'écart souvent important entre les formes postulées à l'origine en proto-mordve et les formes attestées à l'époque moderne. Par exemple, [soda-sask] 'nous les connaissons', du verbe [soda-] <сода> 'savoir', est censée dériver de *soda-saj-n-mek. Comme Kereztes (1999, p. 60) le fait observer, Serebrennikov

postulates such verb-forms whose existence is totally impossible [postule des formes verbales dont l'existence est totalement impossible].

Une première question est d'évaluer la légitimité du patron général à trois composants, et ensuite, vient celle de la forme des composants.

\section{La CàOD avec P1 et P2 (singulier)}

$\mathrm{Au}$ vu de la complexité de la question, il paraît sage de limiter l'analyse dans un premier temps à une partie des formes, et nous commençons l'examen par celles relatives à P1Sg et P2Sg. Dans le tableau, l'amalgame suffixal du présent est indiqué en premier, avec celui du prétérit écrit en dessous :

Tableau 4. Suffixes de la CàOD pour $\mathrm{P} 1$ et $\mathrm{P} 2$

\begin{tabular}{|l|l|l|l|l|l|}
\hline \multicolumn{2}{|l|}{ Mokcha littéraire } & \multicolumn{3}{l|}{ Erzia littéraire } \\
\hline & S1 & S2 & & S1 & S2 \\
\hline 01 & $/$ & -samak-majt & o1 & $/$ & -samak-imik \\
\hline O2 & -te-çten & $/$ & O2 & -tan-itin & $/$ \\
\hline
\end{tabular}

Dans ces suffixes, il semble légitime de reconnaître les pronoms /mon/ P1 et /ton/ P21 . Cela conduit à une première étape de démontage :

-/sodate/ <содате> 'je te connais' : -te- 'P2 objet', sans segment explicitant 'P1 sujet' ou le présent,

•/sodaçten/ <содайхтен> 'je t'ai connu' : -te- 'P2 objet', avec -ç- pour le prétérit et -n pour 'P1 sujet',

-/sodasamak/ <содасамак> 'tu me connais' : -ma- 'P1 objet', - $k$ 'P2 sujet' et -sa- présent,

-/sodamajt/ <содамайть> 'tu m'as connu' : -ma- 'P1 objet', -t ‘P2 sujet' et -j- prétérit.

Dans la langue littéraire mokcha, aucun segment explicite ne correspond à 'P1 sujet' dans /sodate/. Néanmoins, l'erza littéraire et quelques dialectes mokchas présentent 
des formes [sodatan] et [sodaten] qui confirment que /-n/ est le signifiant de 'P1 sujet'. Ces formes livrent une première série d'observations :

1. Le signifiant de 'P2 objet', synchroniquement /-te-/, provient de *hte, comme le montre le maintien d'une occlusive sourde dans [sodate(n)] < * soda-hte-n ainsi que la palatale /ç/ dans [sodaçten] < *soda-j-hte-n. On notera la différence entre P2 -te- < *hte et P5 -da- <*ta. La symétrie actuelle entre P2 /ton/ et P5 /tin/ est trompeuse car les signifiants ne semblent pas avoir la même initiale à l'origine : -hte- pour P2 mais -ta- pour P5.

2. Le signifiant de 'P2 sujet' devait être /-k/. L'erza, avec -k pour le présent et le prétérit, représente très certainement l'état le plus ancien. La forme [sodamajt] du mokcha ne peut résulter que d'une réfection d'un plus ancien *sodamajk, car sinon on devrait trouver ** sodamaçt < *soda-ma-j-ht. Ce morphème $-k$ peut être comparé avec le suffixe d'impératif ${ }^{*}-k$ reconstruit avec le proto-ouralien. Keresztes $(1999$, p. 64$)$ fait observer:

The principle of "archaic heterogeneity" suggests that the Mordvin language is the closest to the original $[\mathrm{PU}]$ protolanguage, with its great variety in its person-marking system. ${ }^{2}$

Si l'on suit cette logique, d'après la CàOD le morphème $-k$ représente 'P2-Sujet' et son utilisation pour l'Impératif est une spécialisation secondaire. Incidemment on peut noter que le youkaghir utilise - $k$ pour le même mode impératif et P2 dans les verbes.

3. On notera que les signifiants ne sont pas les mêmes selon que P1 et P2 sont sujet ou objet.

Tableau 5. Les morphèmes $\mathrm{P} 1$ et $\mathrm{P} 2$

\begin{tabular}{|l|l|l|}
\hline & Objet & Sujet \\
\hline P1 & *-ma- & *-n \\
\hline P2 & *-hte- & *-k \\
\hline
\end{tabular}

1. Le signifiant du prétérit doit être $*_{\mathrm{j}}$ ou $*_{\mathrm{i}}$, ce qui est très cohérent avec les formes mokchas et avec le vocalisme erza au prétérit. Cette situation se retrouve en finnois : ot- $a-n$ 'je prends' $\sim$ ot-i-n 'j'ai pris' avec une opposition : $-a-\sim-i-$.

2. Le signifiant du présent est tantôt $-\varnothing$ - tantôt $-s a-$, venant de *hsa avec maintien de la sourdité. Sur le plan typologique, il est étrange que le présent soit marqué par un segment explicite. Cela incite à postuler que le signifiant le plus probable à date ancienne était l'absence de toute marque : $-\varnothing-$.

30 En conclusion, l'hypothèse d'un patron général à trois composants paraît acceptable mais avec des proto-suffixes différents de ceux proposés par Serebrennikov (1967). De façon assez étrange, cet auteur semble ignorer que P1 et P2 sont exprimés différemment selon qu'ils sont sujet ou objet. Ces considérations amènent aux reconstructions suivantes:

- *soda- Ø-hte-n 'je te connais',

- *soda- Ø-ma-k 'tu me connais',

- *soda-j-hte-n 'je t'ai connu',

- *soda-j-ma-k 'tu m'as connu'.

L'erza semble proche de la situation originale et la principale innovation est d'avoir généralisé le vocalisme /a/ au présent et /i/ au passé. En outre *soda- Ø-ma-k a été refait en *soda-hsa-ma-k dans les deux langues et le segment -hsa- est emprunté à la forme-pivot P3. Le mokcha présente une autre innovation: *soda-ma-j-t qui doit être récent puisque *soda-ma-j-ht est attendu. Cela suggère une chaîne d'innovations : ${ }_{-j}$ ma-k > *-ma-j-k >-ma-j-t avec remplacement de - $k$ P2 par le suffixe plus récent - $t$. 


\section{La CàOD avec P4, P5 et P6 (pluriel)}

P6 étant une forme-pivot pour P4 et P5, ce compartiment de la CàOD offre une bonne transparence. Les tableaux font apparaitre un grand nombre de syncrétismes. En théorie, un inventaire maximal de 28 formes serait possible. En pratique le mokcha littéraire n'en a que 12 et l'erza littéraire 10 , c'est-à-dire que plus de la moitié des formes sont syncrétiques :

Tableau 6. La CàOD en mokcha avec P4/5/6

\begin{tabular}{|l|l|l|l|}
\hline & \multicolumn{4}{|l|}{ Mokcha littéraire } \\
\hline & S6 & S4 & S5 \\
\hline 01 04 & -samaz-maz & $/$ & -samast-mast \\
\hline O2 05 & -tedez-dez & -tedez-dez & $/$ \\
\hline 03 06 & -saz-z & -sask-sk & -sast-st \\
\hline
\end{tabular}

Tableau 7. La CàOD en erza avec P4/5/6

\begin{tabular}{|l|l|l|l|}
\hline & \multicolumn{4}{|l|}{ Erza littéraire } & \\
\hline & S6 & S4 & S5 \\
\hline 01 04 & -samiz-imiz & / & -samiz-imiz \\
\hline O2 05 & -tadiz-idiz & -tadiz-idiz & / \\
\hline 03 06 & -syz-iz & -synek-inek & -synk-ink \\
\hline
\end{tabular}

Plusieurs observations sont possibles:

1. Les suffixes fonctionnent par binôme: en mokcha, les suffixes au présent présentent une syllabe de plus qu'au prétérit : -sa- ou -te-. Cette deuxième forme s'explique sans doute par l'attraction exercée par -tada P5 sur une possible proto-forme *hsa-tez refaite en *-hte-tez > -tedez.

2. En ce qui concerne 'P6 sujet', les deux langues sont cohérentes avec le signifiant ${ }^{*} z$. En mokcha, 'P4 sujet' est $-k$, ce qui est confirmé par l'erzia. 'P5 sujet' en mokcha est -t, cohérent avec/tin/ P5.

3. Quant à 'P6 objet' et 'P3 objet', certaines formes dialectales du mokcha permettent d'y voir plus clair. Au présent, O6S4 -sajnek et 06S5 -saj(e)nt s'opposent à 03S4 -sask et 03S5 -sast. Dans ces formes non syncrétiques, 'P6 objet' est exprimé par -n(e)-.

34 À ce stade de l'analyse, en mettant de côté P3, les différentes briques élémentaires sont les suivantes :

Tableau 8. Les composants élémentaires de la CàOD

\begin{tabular}{|l|l|l|l|l|l|}
\hline & P1 & P2 & P4 & P5 & P6 \\
\hline Sujet & $-\mathrm{n}$ & $-\mathrm{k}$ & $-\mathrm{k}$ & - & $-\mathrm{z}$ \\
\hline
\end{tabular}




\begin{tabular}{|l|l|l|l|l|l|}
\hline Objet & -ma- & -te- $<*$ hte & -ma- & -de- $<*_{\text {te }}$ & -ne- \\
\hline
\end{tabular}

Ce tableau peut maintenant être testé par rapport aux formes que nous n'avons pas encore étudiées. En notant provisoirement 'P3 sujet' par un -X, les formes du mokcha littéraire sont les suivantes :

Tableau 9. La CàOD en mokša avec 06 et S1, S2

\begin{tabular}{|l|l|l|l|l|}
\hline & S1 prévu & S1 réel & S2 prévu & S2 réel \\
\hline 06 & -hsa-ne-n-j-ne-n & -sa-j-ne-j-ne & -hsa-ne-k-j-ne-k & -sa-j-t-j-t \\
\hline
\end{tabular}

Dans les dialectes, on rencontre des formes plus proches de notre reconstruction. Dans le sud-est du domaine mokša, on trouve -inen pour 'O6S1 prétérit' et -jenk pour 'O6S2 prétérit'. Ces formes sont logiquement les plus anciennes.

Tableau 10. La CàOD en mokcha avec 06 et S3

\begin{tabular}{|l|l|l|}
\hline & S3 prévu & S3 réel \\
\hline O6 & -hsa-ne-X-j-ne-X & -syne-zen \\
\hline
\end{tabular}

La forme 'O6S3 prétérit' -zen est insolite mais en erza et plus sporadiquement en domaine mokcha on trouve le suffixe -inze, qui doit représenter un état plus ancien sans métathèse. Cela amène à supposer que le suffixe $\mathrm{X}=$ 'P3 sujet' est $-z e<{ }^{*}$-se. 'O6S3 présent' -syne ne semble pas présenter ce segment, mais ici encore, en erza et en mokcha dialectal, on trouve -synze. Le démontage que nous proposons permet de déterminer dans le maquis des formes dialectales celles qui sont réellement anciennes et « logiques », c'est-à-dire prévisibles.

On peut également constater qu'en mokcha le signifiant $-j$ - du prétérit s'est infiltré dans les suffixes relatifs au présent, par fausse coupe, ce qui achève la ruine de l'ancien système temporel $-\varnothing-\sim-j$ - au profit du nouveau système $-s a-\sim-\emptyset$-. L'erza a développé une autre innovation $-(s) a^{-} \sim-i-$. De façon générale, la logique de chacune des langues mordves est que le mokcha tend à opposer des segments courts à des segments longs alors que l'erza s'appuie plutôt sur le vocalisme pour distinguer le présent du prétérit.

\section{La CàOD avec P3}

Il reste à examiner les formes où P3 est sujet ou objet. Deux séries de formes ont déjà été décrites ci-dessus dans le tableau 9.

Tableau 11. La CàOD avec S3

\begin{tabular}{|l|l|l|l|}
\hline & Proto-mordve & Mokcha & Erza \\
\hline & S3 prévu & S3 réel & S3 réel \\
\hline 04 & *-hsa-ma-se*-j-ma-se & -sa-ma-z-ma-z & -sa-mi-z-i-mi-z \\
\hline
\end{tabular}




\begin{tabular}{|l|l|l|l|}
\hline o5 & *hsa-te-se*-j-te-se & -te-de-z-de-z & -ta-di-z-i-di-z \\
\hline
\end{tabular}

Lorsque P5 est objet, le signifiant du présent est -te-/-ta- au lieu de -sa-, sous la double attraction de -tada où -ta- est issu du pluriel nominal et du pronom /tin/. D'autre part, le syncrétisme a abouti à l'élimination de la voyelle finale -e, comme dans 04S6 et 05S6. La suite des formes concerne P1 et P2 objet:

Tableau 12. La CàOD avec S3

\begin{tabular}{|l|l|l|l|}
\hline & Proto-mordve & Mokcha & Erza \\
\hline & S3 prévu & S3 réel & S3 réel \\
\hline 01 & *-hsa-ma-se*-j-ma-se & -sa-ma-n-ma-n & -sa-ma-m-i-mi-m \\
\hline 02 & *-(hsa)-hte-se*-j-hte-se & -ta-nza-nze & -ta-nza-t-i-nzi-t \\
\hline
\end{tabular}

41 Les formes attendues pour 01S3 auraient été presque homophones de 04S3. Par une sorte de pléonasme suffixal, un signifiant relatif à P1 apparaît deux fois dans la construction de 01S3. Les formes relatives à $02 \mathrm{~S} 3$ sont profondément remaniées. En erza, la logique de pléonasme suffixal aboutit à -ta-nza-t où $\mathrm{P} 2$ est, en pratique, suffixé deux fois. Le suffixe *-se >-ze a été étoffé en -nze/-nza d'après la flexion pronominale / tej-nza/ <тейнза> 'à lui' ${ }^{3}$. Comme dans le cas de 02P1 *-hte(n), -sa- est absent, de sorte que c'est curieusement 'P2 objet' *-hte- qui a été réinterprété comme le signifiant du présent. Cette troncation aboutit à une forme paradoxale surcourte -nze, dépourvu de segment relatif à P2.

Il faut maintenant examiner les formes où $\mathrm{P} 3$ est objet :

Tableau 13. La CàOD avec 03 et $\mathrm{S} 1$

\begin{tabular}{|l|l|l|l|}
\hline & Proto-Mordve & Mokcha & Erza \\
\hline & S1 prévu & S1 réel & S1 réel \\
\hline 03 & *-hsa-n*-j-n & -sa-j-ne & -sa-i-ja \\
\hline
\end{tabular}

La nasale finale attendue pour 03S1 -san est attestée uniquement en erzia méridional.

Tableau 14. La CàOD avec 03 et S2

\begin{tabular}{|l|l|l|l|}
\hline & Proto-Mordve & Mokcha & Erza \\
\hline & S2 prévu & S2 réel & S2 réel \\
\hline O3 & *-hsa-k*-j-k & -sa-k-j-te & -sa-k-i-k \\
\hline
\end{tabular}

En mokcha, au prétérit, la finale - $k$ a été remplacée à date récente par -te analogique de /ton/ comme dans soda-majt 'tu m'as connu'. L'absence de dévoisement de -jte, pour *ihte qui serait attendu, en témoigne. 


\section{Le cas particulier de la CàOD avec P3 objet et sujet}

On touche ici au cœur du système de la CàOD tel qu'il a pu se mettre en place en protomordve. Et il fallait clarifier la formation de tous les autres suffixes avant d'aborder ceux-ci. Comme il a été souligné précédemment un système verbal où le présent est exprimé par un segment explicite est typologiquement rare. Et ce point est d'autant plus étonnant que le prétérit en mordve était doté d'un signifiant propre ${ }^{*}{ }_{-i}$ - . Le tableau des formes est le suivant :

Tableau 15. La CàOD avec 03 et S3

\begin{tabular}{|l|l|l|l|}
\hline & Proto-mordve & Mokcha & Erza \\
\hline & S3 prévu & S3 réel & S3 réel \\
\hline O3 & *-hsa-se*-j-se & -sy-ze & -sy-i-ze \\
\hline
\end{tabular}

Le suffixe long 03P3 $*$-saze $<$ *hsa-se est attesté en erza dialectal nord sous la forme sazo, avec réfection harmonique $*_{-} \mathrm{e}>-0$. Le suffixe court -sy peut être interprété comme une haplologie *hsa-se $>$ *ssy $>-s y$, avec un vocalisme assez inattendu, dont la motivation est peut-être d'éviter toute confusion avec celui des autres suffixes. On peut en outre noter que le suffixe -sy <-сы> est prononcé [səj] par plusieurs locuteurs dont nous avons des enregistrements.

47 À ce stade de l'analyse on aborde la question de l'état initial du système proto-mordve. Deux options sont possibles : (1) *-hsa- est le signifiant du présent « depuis le début ", ou (2) *-hsa- était en fait le signifiant de 'P3 objet'. Nous pensons que la deuxième est meilleure et la deuxième partie de l'article est consacrée à la défendre.

Outre la rareté typologique d'un présent explicite, il existe une solidarité manifeste entre certains suffixes de la CàOD et les pronoms anaphoriques:

Tableau 16. Les anaphoriques

\begin{tabular}{|c|c|c|}
\hline Mokcha & P3 & P6 \\
\hline Anaphoriques & sä <ç> celui-là & nä-t < $<$ HЯT> ceux-ci \\
\hline Article défini (suffixé) & $-\mathrm{s}<-\mathrm{cb}>\mathrm{le}, \mathrm{la}$ & -t-ne $<$ THe $>$ les \\
\hline Pronoms personnels & son $<\mathrm{COH}>$ il, elle & sin $<$ синь $>$ ils, elles \\
\hline Signifiants de la COD & *-hsa- <-ca-> 03 & $*$-ne- $<$ He $>06$ \\
\hline
\end{tabular}

Sur le plan comparatif, on peut noter que le « hasard» de la ressemblance en mordve des formes listées dans le tableau 14 a son pendant exact en français: /son/ $<\mathrm{coH}>i l$, elle /-sí/ <сы> le, la /-ś/ <-сь> le, la. Ces mots français dérivent d'un mot latin unique : illus, illa comme en italien, espagnol, etc. L'hypothèse discutée dans la suite est que les suffixes /-sí, -səj/ <cы>, *-hsa- <-ca-> et le pronom autonome /son/ < $\mathrm{coH}>$ sont génétiquement liés et ne sont en réalité qu'une seule et même unité lexicale dans des emplois syntaxiques différents. La question se pose maintenant de la genèse du système. 


\section{Les anaphoriques}

Les anaphoriques et les déictiques ont été beaucoup étudiés par les théories de l'énonciation et peut-être moins par les théories qui se concentrent principalement sur la description des langues. Nous souhaitons éclairer un aspect des anaphoriques qui semble avoir été peu décrit jusqu'à présent : leur participation dans les processus de grammaticalisation. Le cas de la CàOD en mordve est à notre avis tout à fait exemplaire.

51 En introduction de l'article, nous avons défini les notions de CàOD ou CàOI à partir de deux énoncés en français : il mange de la viande $\sim$ il mange la viande, le premier étant un exemple de CàOI et le second de CàOD. Il est possible de pronominaliser ces deux exemples : il en mange (CàOI) il la mange (CàOD). De façon pléonastique, on peut même dire : il en mange, de la viande $\sim$ il la mange, la viande.

Par rapport au français, nous proposons d'analyser la CàOD en mordve comme s'il était obligatoire de dire il la-mange la viande. La suppression de l'anaphorique <la>, correspondant au segment *hsa du proto-mordve *hsa-se > /si/ <cы>, est possible en français, et même souhaitable stylistiquement, alors qu'elle est devenue impossible en mordve. Autrement dit, dans cette optique, la CàOD a pour origine le figement et la grammaticalisation de ce qui était initialement un pléonasme. Le retour vers la forme simple il mange la viande n'est plus possible dans la synchronie actuelle du mokcha et de l'erza. Notre approche est que le signifiant *hsa, initialement celui de l'anaphorique le, la, a été réinterprété de façon irréversible comme étant celui du présent lors de la genèse de la CàOD en proto-mordve. L'ensemble des formes du tableau 14 ne laisse guère de doute sur la validité de cette analyse. Le démontage de l'ensemble des formes dialectales et la logique de ce système sont autant d'indications favorables.

Dans cet article, nous avons établi de proche en proche les différents morphèmes utilisés en proto-mordve pour la CàOD. Ils sont récapitulés dans les tableaux suivants et ils peuvent à leur tour faire l'objet d'une analyse plus profonde :

Morphèmes pronominaux singuliers

\begin{tabular}{|l|l|l|l|}
\hline & P1 & P2 & P3 \\
\hline Pronom libre & mon & ton & son \\
\hline Suffixe objet & -ma- & *-hte- & *-hsa- \\
\hline Suffixe sujet & -n & -k & *-se \\
\hline
\end{tabular}

Morphèmes pronominaux pluriels

\begin{tabular}{|l|l|l|l|}
\hline & P4 & P5 & P6 \\
\hline Pronom libre & min & tin & sin \\
\hline Suffixe objet & -ma- & *-te- & -ne- \\
\hline Suffixe sujet & -k & -t & *s \\
\hline
\end{tabular}


54

Certains suffixes ont subi des réinterprétations étonnantes. L'anaphorique *hsa a été, de bonne heure, utilisé comme signifiant du Présent, spécialement pour P1, P3, P4 et P6. Le suffixe de pluriel nominal a envahi le paradigme verbal en tant que signifiant du présent pour P4 et P5. De façon générale, on peut observer que l'équilibre général d'un corpus de formes aussi complexe que la morphologie verbale du mordve est difficile à préserver quand un grand nombre de morphèmes peuvent être interprétés de plusieurs façons. La présence d'un segment fricatif vélaire dans *-hte- et *-hsa- peut être comparé au marqueur de présent en vogul-mansi $-\gamma-$. Dans ce cas, si on accepte d'y voir un morphème cognat, ces segments peuvent être analysés plus avant comme étant ${ }^{*}-\gamma$-teet $*-\gamma$-sa-.

Morphèmes de temps verbal de la CàOD

\begin{tabular}{|l|l|l|l|}
\hline & POu & Erza & Mokcha \\
\hline Présent & $-\varnothing--\gamma-$ & $-(\mathrm{s})-\mathrm{a}-(\mathrm{t})-\mathrm{a}$ & $-\mathrm{s}-\mathrm{-t}-$ \\
\hline Passé & $-\mathrm{i}-$ & $-\mathrm{i}-$ & $-\varnothing-$ \\
\hline
\end{tabular}

Par ailleurs, on peut noter que les suffixes objet sont dans plusieurs cas identiques aux suffixes de la prédication nominale et de la CàOI, en particulier ceux de P2, P4 et P5. Cette structure est typique d'un système ergatif : le sujet intransitif et l'objet d'un verbe transitif ont la même forme. In fine, si l'on accepte le jugement de Keresztes sur l'ancienneté proto-ouralienne de la situation reflétée en mordve cela débouche sur l'hypothèse que le proto-ouralien présentait à l'origine un système de type ergatif et non pas accusatif.

\section{BIBLIOGRAPHIE}

ABONDOLo Daniel M. (ed), 1998, The Uralic Languages, London and New York: Routledge.

AHLQUIST A., 1861, Versuch einer moksha-mordwinischen Grammatik nebst Texten und Wörterverzeichniss, Saint-Petersbourg : Eggers et Comp.

ALJAMКIN 2000 = АЛЯМКИН Н.С., МОКШЕН КЯЛ : МОРФОЛОГИЯ ; ВУЗОНЬ МОКШЕН И ФИННО-УГРАНЬ ОТДЕЛЕНИЯНЬ ТОНАФНИХНЕНДИ УЧЕБНИК, САРАНСК : КРАСНЫЙ ОКТЯБРЬ.

BOMHARD A., 2008, Reconstructing Proto-Nostratic, Comparative Phonology, Morphology, And Vocabulary. 2M vol. Leiden: Brill.

BUBRIH 1953 = БУБРИХ Д.В., ИСТОРИЧЕСКАЯ ГРАММАТИКА ЭРЗЯНСКОГО ЯЗЫКА, САРАНСК :МОРДОВСКОЕ КНИЖНОЕ ИЗДАТЕЛЬСТВО.

BUDENZ József, 1876, Mordwinische Grammatik, Budapest.

Études finno-ougriennes, 46 | 2014 
BUDENZ József, 1899, Moksa- és erza-mordvin nyelvtan, Budapest.

COLLINDER Björn, 1955, Fenno-Ugric Vocabulary: An Etymological Dictionary of the Uralic Languages, Stockholm: Almqvist \& Viksell. (1977 Second, revised edition: Hamburg: Helmut Buske Verlag.) COLLINDER Björn (ed.), 1957, Survey of the Uralic Languages, Stockholm: Almquist \& Wiksell. COLLINDER Björn, 1960, Comparative Grammar of the Uralic Languages, Stockholm: Almquist \& Wiksell. COLLINDER Björn (ed.), 1965, An Introduction to the Uralic Languages, Berkeley: University of California Press.

DÉcsY Gyula, 1990, The Uralic Protolanguage: A Comprehensive Reconstruction, Bloomington, Indiana. DONNER K., 1944, Kamassisches Wörterbuch, Helsinki : Suomalais-Ugrilainen Seura.

FOURNET Arnaud, 2008, «Le vocabulaire mordve de Witsen : une forme ancienne du dialecte zubumokša », Études Finno-Ougriennes, vol 40, p. 57-84.

FOURNET Arnaud, 2010, Le moksha, une langue ouralienne, Saarbrücken : Editions Universitaires Européennes.

FOURNET Arnaud, 2011, «Quelques éléments historiographiques concernant la famille ouralienne aux XVII ${ }^{\mathrm{e}}$ et XVIII ${ }^{\mathrm{e}}$ siècles », Beiträge zur Geschichte der Sprachwissenchaft, vol. 2,1, p. 55-64.

FOURNET Arnaud, 2011, "About the difficulty of determining the lexical classes of the Moksha language”, ReVEL, vol. 9.17, p. 33-54.

HAJDÚ Péter, 1963, Finnugor népek és nyelvek, Budapest : Gondolat kiadó.

HAJDÚ Péter, 1975, Finno-Ugrian Languages and Peoples, traduit par G. F. CUSHING. London : André Deutsch.

HERRALA E. \& FEOKTISTOV A., 1998, Mokshalais-suomalainen sanakirja. Turku : Publications of the Department of Finnish and General linguistics of the University of Turku (48).

KERESZTES László, 1999, Development of Mordvin Definite Conjugation, Helsinki, Suomalais-Ugrilainen Seura, Mémoires de la société finno-ougrienne, $n^{\circ} 233$.

KURYLOWICZ Jerzy, 1945-49, « La nature des procès dits analogiques », Acta Linguistica 5, p. 15-37. LAAKSO Johanna, 1992, Uralilaiset kansat, Porvoo-Helsinki-Juva.

REDÉl K. (éd.), 1986-88. Uralisches etymologisches Wörterbuch, 3 volumes. Budapest, Akadémiai kiadó. SAMmallahti Pekka, 1988, "Historical phonology of the Uralic Languages", in Sinor, Denis (ed.), The Uralic Languages, Leiden: E.J. Brill, p. 478-554.

SEREBRENNIKOV 1967, = СЕРЕБРЕННИКОВ В.А., ИСТОРИЧЕСКАЯ МОРФОЛОГИЯ МОРДОВСКИХ ЯЗЫКОВ, МОСКВА.

SEREBRENNIKOV, FEOKTISTOV \& POLJAKOVA 1998 = СЕРЕБРЕННИКОВ В.А., ФЕОКТИСТОВ А.П., ПОЛЯКОВА О.Е. (РЕД.), МОКШАНСКО-РУССКИЙ СЛОВАРЬ, МОСКВА : ИЗДАТЕЛЬСТВО РУССКИЙ ЯЗЫК.

STAROSTIN S., A.V. DYBO \& O.A. MUDRAK. 2003, Etymological Dictionary of the Altaic Languages, Leiden: Brill.Zaicz G., 1998, « Mordva » [en fait surtout l'erzia], ABONDOLO, D. (ed.), The Uralic Languages, London and New York: Routledge. 


\section{NOTES}

1. Les personnes sont numérotées de 1 à 6 dans l'article. P1pluriel, P2pluriel et P3pluriel sont donc P4, $\mathrm{P} 5$ et $\mathrm{P} 6$ respectivement.

2. Le principe de l'hétérogénéité archaïque suggère que le mordve est le plus proche de la protolangue [ouralienne] originale, avec la grande variété de son système de désinences pronominales.

3. À noter au passage cette forme, isolée, qui va dans le sens de l'approche autrefois proposée par J. Perrot.

\section{RÉSUMÉS}

Plusieurs langues ouraliennes, comme le hongrois, le mokcha ou l'erza, possèdent une double série de désinences verbales suivant que le complément d'objet est défini ou indéfini, ce que la tradition ouralistique appelle conjugaison objective définie ou indéfinie, ou plutôt à objet défini (CàOD) ou indéfini (CàOI). L'article décrit les différentes séries de suffixes pronominaux en mokcha, en comparaison avec l'erza, et s'efforce de démonter les suffixes de la CàOD afin d'en déterminer les composants élémentaires. L'hypothèse développée ensuite pour expliquer l'émergence de la CàOD en mordve est que certains pronoms anaphoriques ont été grammaticalisés de façon irréversible en tant que marque de temps.

\section{INDEX}

Index géographique : Fédération de Russie, Moscou

disciplines erza, langues mordves, mokcha

Mots-clés : anaphore, anaphore, conjugaison, conjugaison objective, conjugaison objective, morphologie, déictiques, déictiques

Keywords : Anaphora, Conjugation, Deictics, Morphology, Objective Conjugation

Thèmes : linguistique 